\title{
ON TANNAKA-TERADA'S PRINCIPAL IDEAL THEOREM FOR RATIONAL GROUND FIELD
}

\author{
SHÔICHI TAKAHASHI
}

Let $n$ be a natural number $2 \nmid n$ or $4 \mid n$ and $m$ be one more natural number which have no quadratic factor and satisfy the relation $Q\left(\zeta_{n}\right) \supset Q(\sqrt{ } m)$ $\left(Q:\right.$ the rational number field, $\left.\zeta_{n}=\exp (2 \pi i / n)\right)$, then the author wants to give an explicit representation for Tannaka-Terada's principal ideal theorem for the case of $Q\left(\zeta_{n}\right) \supset Q(\sqrt{m}) \supset Q$. In 1 we express the calculation of Geschlechtermodul $\mathfrak{F}_{n}$ of $Q\left(\zeta_{n}\right) / Q$ and $\mathfrak{M}=\mathrm{f}\left(Q\left(\zeta_{n}\right) / Q(\sqrt{m}) / Q\right)$ according to the definition and notation of T. Tannaka [1], S. Takahashi [5]. In 2 we show that the ideals in each ambigous ideal class mod. $\mathfrak{M}$ which are prime to $n$ (i.e. $\mathscr{N}$ an ambigous ideal in $Q(\sqrt{m})$ prime to $n$ satisfying the relation $\mathfrak{P}^{\sigma-1}=(\alpha), \alpha \in Q(\sqrt{m}), \alpha \equiv 1(\bmod . \mathfrak{M})$ there $\sigma$ means a generator of the Galois group of $Q(\sqrt{m}) / Q)$ ), are only principal $\mathfrak{A}=(A)$ ideals in $Q(\sqrt{m})$, and decide their form explicitly. In 3 it is shown that we can find a unit $E(A)$ in $Q\left(\zeta_{n}\right)$ explicitly, for which

$$
A \equiv E(A) \quad\left(\bmod . \mathfrak{F}_{n}\right)
$$

so that

$$
\mathfrak{A} \sim 1\left(\bmod . \mathfrak{F}_{n}\right) \text { in } Q\left(\zeta_{n}\right)
$$

holds.

1. Calculation of $\mathfrak{F}_{n}, \mathfrak{M}$. Let $n=p_{1}^{e_{1}} p_{2}^{e_{2}} \cdots p_{t}^{e_{t}}$ be a natural number, where $p_{1}, p_{2}, \cdots, p_{t}$ are different prime numbers and $p_{1}=2, e_{1}=0$ or $e_{1} \geqq 2$, and $\mathfrak{F}_{n}$ the "Geschlechtermodul" of $Q\left(\zeta_{n}\right) / Q$. We have then from S. Takahashi [5]

$$
\mathfrak{F}_{n}=\mathfrak{F}_{p_{1}} \mathfrak{F}_{p_{2}} \cdots \mathfrak{F}_{p_{t}}, \quad \mathfrak{F}_{p_{\mathrm{s}}}=\left(1-\zeta_{p_{i}}\right), \quad i=1,2, \cdots, t
$$

Subsequently, let $\mathrm{f}\left(Q\left(\zeta_{n}\right) / Q\right)$ and $\mathrm{f}(Q(\sqrt{m}) / Q)$ be "Fühlers" of $Q\left(\zeta_{n}\right) / Q$ and $Q(\sqrt{m}) / Q$ respectively, then

$$
\begin{aligned}
& \mathrm{f}\left(Q\left(\zeta_{n}\right) / Q\right)=n p_{\infty} \\
& \mathrm{f}(Q(\sqrt{m}) / Q)=d p_{\infty}{ }^{e}= \begin{cases}m p_{\infty}{ }^{e} & (m \equiv 1(\bmod .4)) \\
4 m p_{\infty}{ }^{e} & (m \equiv 2,3(\bmod .4))\end{cases}
\end{aligned}
$$


(provided that $e=0$ for $m>0, e=1$ for $m<0$ ) hold.

Now from $Q\left(\zeta_{n}\right) \supset Q(\sqrt{m})$ we have $|d| \mid n$, and set $n=|d| n^{\prime}$. Therefore, according to the definitions and notations of $T$. Tannaka [1], we get

$$
\mathfrak{M}=\mathrm{f}\left(Q\left(\zeta_{n}\right) / Q(\sqrt{m}) / Q\right)=\mathfrak{D}\left(Q\left(\zeta_{n}\right) / Q(\sqrt{m})\right) \cdot \mathfrak{F}\left(Q\left(\zeta_{n}\right) / Q\right) .
$$

On the other hand

$$
\mathfrak{D}\left(Q\left(\zeta_{n}\right) / Q(\sqrt{m})\right) \mathfrak{D}(Q(\sqrt{m}) / Q)=\mathfrak{D}\left(Q\left(\zeta_{n}\right) / Q\right)
$$

hence

$$
\begin{aligned}
\mathfrak{M} & =\mathfrak{D}\left(Q\left(\zeta_{n}\right) / Q\right) \mathfrak{F}\left(Q\left(\zeta_{n}\right) / Q\right) / \mathfrak{D}(Q(\sqrt{m}) / Q) \\
& =f\left(Q\left(\zeta_{n}\right) / Q\right) / \mathfrak{D}(Q(\sqrt{m}) / Q) \\
& =n p_{\infty} / \sqrt{d} p_{\infty}^{e} \\
& =n^{\prime} \sqrt{d} p_{\infty}^{e^{\prime}}
\end{aligned}
$$

(provided that $e^{\prime}=0$ for $m<0$ and $e^{\prime}=1$ for $m>0$ ).

From the above, we get the following proposition.

PROPOSITION 1. Let $m, n$ be as above and $Q\left(\zeta_{n}\right) \supset Q(\sqrt{m}) \supset Q$, then

$$
\begin{aligned}
& \mathfrak{F}_{n}=\mathfrak{F}\left(Q\left(\zeta_{n}\right) / Q\right)=\mathfrak{F}_{p_{1}} \mathfrak{F}_{p_{2}} \cdots \mathfrak{F}_{p_{t}}, \quad \widetilde{F}_{p_{t}}=\left(1-\zeta_{p_{t}}\right) \\
& \mathfrak{M}=\mathfrak{f}\left(Q\left(\zeta_{n}\right) / Q(\sqrt{m}) / Q\right)=n p_{\infty} / \sqrt{d} p_{\infty}^{e} \\
&= n^{\prime} \sqrt{d} p_{\infty}^{e^{\prime}},
\end{aligned}
$$

provided that

$$
\begin{aligned}
& d= \begin{cases}m & (m \equiv 1(\bmod .4)) \\
4 m & (m \equiv 2,3(\bmod .4))\end{cases} \\
& n^{\prime}=n /|d|, \quad e^{\prime}=\left\{\begin{array}{lll}
1, & \text { for } & m>0 \\
0, & \text { for } & m<0 .
\end{array}\right.
\end{aligned}
$$

2. A decision of ambigous ideals mod $\mathfrak{M}$. Let $\sigma$ be the generator of the Galois group of $Q\left(\zeta_{n}\right) / Q$ such that $\sqrt{m^{\sigma}}=-\sqrt{m}$, and $\mathfrak{A}$ an ambigous ideal mod. $M$ prime to $n$, then

$$
\mathfrak{A}^{\sigma-1}=(\alpha), \quad \mathfrak{A}^{\sigma}=(\alpha) \mathfrak{R}, \quad \alpha \in Q(\sqrt{m}), \quad \alpha \equiv 1(\bmod . \mathfrak{M}) .
$$

Here we set 


$$
\alpha=\frac{\lambda}{\mu} \quad \lambda, \mu \text { are integers of } Q(\sqrt{m}) \text { prime to } n \text {. }
$$

Now from $\alpha \equiv 1(\bmod . \mathfrak{M})$

$$
\begin{aligned}
\lambda-\mu & \equiv 0 \quad(\bmod . \mathfrak{M}) \\
\frac{\alpha+1}{2}-1 & =\frac{\alpha-1}{2}=\frac{\lambda-\mu}{2 \mu}
\end{aligned}
$$

hold. On the other hand, from $\mathfrak{A}^{\sigma-1}=(\alpha)$ we get

$$
N \alpha= \pm 1 \quad(N: \text { the norm } Q(\sqrt{m}) \rightarrow Q)
$$

here

$$
\begin{array}{lr}
\text { if } m<0, & N \alpha>0 \\
\text { if } \quad m>0, \text { from } \alpha \equiv 1\left(\bmod . p_{\infty}\right) & N(\alpha)>0
\end{array}
$$

hold. Therefore, for any cases we can set

$$
N \alpha=1, \quad \alpha^{\sigma}=1 / \alpha .
$$

Now from

$$
\left(\frac{\alpha+1}{2} \mathfrak{A}\right)^{\sigma}=\frac{\alpha^{\sigma}+1}{2} \mathfrak{U} \sigma=\frac{1 / \alpha+1}{2} \cdot \alpha \mathfrak{A}=\frac{\alpha+1}{2} \mathfrak{A}
$$

$\frac{\alpha+1}{2} \mathfrak{A}$ is an $\sigma$-invariant ideal of $Q(\sqrt{m})$ which is not always prime to $n$. Therefore, if we set all prime numbers in $d, p_{1}, p_{2}, \cdots, p_{t}$ and $p_{i}=\mathfrak{p}_{i}^{2}$ in $Q(\sqrt{m})$, then we get

$$
\frac{\alpha+1}{2} \mathfrak{A}=(a) \mathfrak{p}_{1}^{\lambda_{1}} \mathfrak{p}_{2}^{\lambda_{2}} \cdots \mathfrak{p}_{t}^{\lambda_{t}}
$$

(provided that $a$ is a rational number, where $\lambda_{i}=0$ or 1 ).

In the following lines we decide $\mathfrak{A}$ for each case of $m$ (mod.4).

I. $m \equiv 1(\bmod .4)$

In this case, $d=m$ is prime to 2 , and $n$ is prime to 2 or $4 \mid n$. Therefore from 1 , proposition 1 we get

$\mathfrak{M}$ is prime to 2 or $4 \mid \mathfrak{M}$ and

$\mathfrak{F}_{n}$ is prime to 2 or $2 \| \mathfrak{M}$.

If $n$ is prime to 2 , then so is $\mathfrak{M}$. Hence from (2), (3) we get 


$$
\frac{\alpha+1}{2} \equiv 1 \quad(\bmod . \mathfrak{M})
$$

and from $\mathfrak{F}_{n} \mid \mathfrak{M}$,

$$
\frac{\alpha+1}{2} \equiv 1 \quad\left(\bmod . \mathfrak{F}_{n}\right)
$$

holds.

If $4 \mid n$, then $4 \mid \mathfrak{M}, 2 \| \mathfrak{F}_{n}$. Let $\mathfrak{M}^{\prime}$ be the maximal part of $\mathfrak{M}$ relatively prime to $n$, then as above

$$
\frac{\alpha+1}{2} \equiv 1 \quad\left(\bmod . \mathfrak{M}^{\prime}\right)
$$

holds.

Furthermore, from $2 \| 2 \mu, \lambda-\mu \equiv 0(\bmod .4)$ and (3), we get

$$
\frac{\alpha+1}{2} \equiv 1 \quad(\bmod .2) \text {, }
$$

therefore from $\mathfrak{F}_{n} \mid(2) \mathfrak{M}^{\prime}$

$$
\frac{\alpha+1}{2} \equiv 1 \quad\left(\bmod . \mathfrak{F}_{n}\right)
$$

Thus we have the following proposition.

PROPOSITION 2. Let $m$ be $m \equiv 1$ (mod. 4) and $\mathfrak{A}$ an ideal of an ambigous ideal class mod. $\mathfrak{M}$ in $Q(\sqrt{m}) / Q$, i.e.

$$
\mathfrak{A}^{\sigma-1}=(\alpha), \alpha \in Q(\sqrt{m}), \alpha \equiv 1(\bmod . \mathfrak{M})
$$

then

$$
\frac{\alpha+1}{2} \equiv 1 \quad\left(\bmod . \mathfrak{F}_{n}\right)
$$

and $\frac{\alpha+1}{2}, \mathfrak{A}$ are both prime to $n$ and $d$, now from (4) we have

$$
\frac{\alpha+1}{2} \mathfrak{U}=(a), \text { a is a rational number prime to } n
$$

and

$$
\begin{gathered}
\mathfrak{U}=\left(\frac{a}{\left.\frac{\alpha+1}{2}\right)} \text { is principal in } Q(\sqrt{m})\right. \\
\frac{a}{\frac{\alpha+1}{2}} \equiv a\left(\bmod . \mathfrak{F}_{n}\right)
\end{gathered}
$$


II. $m \equiv 3(\bmod .4)$

In this case, $d=4 m p_{\infty}^{e}, 4|n, 2| \mathfrak{M}$. For the maximal part $\mathfrak{M}^{\prime}$ of $\mathfrak{M}$ which is prime to $n$, as by the case of $\mathbf{I}$, we get

$$
\frac{\alpha+1}{2} \equiv 1 \quad\left(\bmod . \mathfrak{M}^{\prime}\right)
$$

For mod. 2, we set $(2)=\mathfrak{p}^{2}$ in $Q(\sqrt{m}), \mathfrak{p}=(2,1+\sqrt{m})$ and investigate it corresponding to the following cases.

i) $\mathfrak{p}^{2 k} \| \frac{\alpha+1}{2}, \quad k=0,1,2, \cdots$

Then $\frac{\alpha+1}{2^{k+1}}$ is prime to 2 and from

$$
\left(\frac{\alpha+1}{2^{k+1}}\right)^{\sigma} \mathfrak{A}^{\sigma}=\frac{\alpha+1}{2^{k+1}} \mathfrak{A}
$$

$\frac{\alpha+1}{2^{k+1}} \mathfrak{A}$ is prime to $n$ especially to $d$. Therefore $\left(\frac{\alpha+1}{2^{k+1}}\right) \mathfrak{A}$ is a $\sigma$-invariant ideal prime to $d$. Now from (4), we have

$$
\frac{\alpha+1}{2^{k+1}} \mathfrak{A}=(a), \quad a \text { is a rational number prime to } n
$$

and

$$
\mathfrak{A}=\left(\frac{a}{\frac{\alpha+1}{2^{k+1}}}\right) \text { is principal in } Q(\sqrt{m})
$$

ii) $\mathfrak{p}^{2 k+1} \| \frac{\alpha+1}{2}$

Then $\mathfrak{p} \| \frac{\alpha+1}{2^{k+1}}$ holds. And we can set

$$
\begin{aligned}
& \beta=\frac{\alpha+1}{2^{k+1}}=\frac{\beta_{0}}{b} \\
& \beta_{0}=x+y \sqrt{m}
\end{aligned}
$$

provided that $x, y, b$ are rational integers, $b$ is prime to $n$, and $\beta_{0}$ is an integer in $Q(\sqrt{m})$ satisfying the condition $\mathfrak{p} \| \beta_{0}$.

Now from $\mathfrak{p}=(2,1+\sqrt{m}), \mathfrak{p} \| \beta_{0}, x, y$ must be both odd numbers, because if $x, y$ are both even then $2 \mid \beta_{0}$, if $x$ is odd, and $y$ is even i.e. $x=2 s+1$ $y=2 t$ ( $s, t$ are rational integers), then from 


$$
\beta_{0}=2 s+1+2 t \sqrt{m}=2(s+t \sqrt{m})+1, \mathfrak{p} \nmid \beta_{0}
$$

and if $x$ is even, $y$ is odd, i.e. $x=2 s, y=2 t+1(s, t$ are rational integers $)$ then from

$$
\begin{aligned}
\beta_{0} & =2 s+(2 t+1) \sqrt{m} \\
& =2(s+t \sqrt{m})+\sqrt{m}, \quad \mathfrak{p} \nmid \beta_{0} .
\end{aligned}
$$

We have then

$$
\beta_{0}^{1-\sigma}=(\alpha+1)^{1-\sigma}=\alpha \equiv 1 \quad(\bmod . \mathfrak{M})
$$

especially

$$
\beta_{0}^{1-\sigma} \equiv 1 \quad(\bmod .2)
$$

On the other hand we have

$$
\begin{gathered}
\beta_{0}^{1-\sigma}-1=\frac{2 y \sqrt{m}}{x-y \sqrt{m}} \\
\mathfrak{p}^{2}\|2 y \sqrt{m}, \mathfrak{p}\| x-y \sqrt{m}, \\
\beta_{0}^{1-\sigma} \neq 1 \quad(\bmod .2) .
\end{gathered}
$$

Therefore the case ii) does not happen. As was stated above, we have the following proposition.

Proposition 2'. Let $m$ be $m \equiv 3(\bmod .4)$ and $\mathfrak{A}$ an ambigous ideal of an ambigous ideal class mod. $\mathfrak{M}$ in $Q(\sqrt{m}) / Q$ i.e. $\mathfrak{A}^{\sigma-1}=(\alpha), \alpha \in Q(\sqrt{m})$,

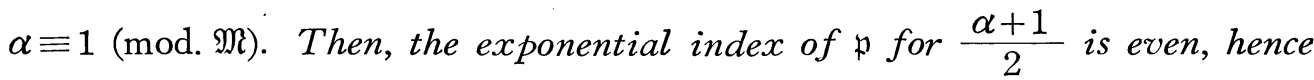
we can set $\mathfrak{p}^{2 k} \| \frac{\alpha+1}{2}(k=0,1,2, \cdots)$. And $\frac{\alpha+1}{2^{k+1}} \mathfrak{A}$ is a $\sigma$-invariant ideal of $Q(\sqrt{m})$ prime to $n$. Therefore again from (4), we get

$$
\frac{\alpha+1}{2^{k+1}} \mathfrak{A}=(a), \quad \mathfrak{U}=\left(\frac{a}{\frac{\alpha+1}{2^{k+1}}}\right) \text { is principal in } Q(\sqrt{m})
$$

$a$ is a rational number prime to $n$.

\section{III. $m \equiv 2(\bmod .4)$}

In this case, we have $d=4 m p_{\infty}^{e}, n=2^{t} \cdot n_{0}\left(t \geqq 3, n_{0}\right.$ odd). And if we set $2=\mathfrak{p}^{2}$ in $Q(\sqrt{m})$, then 


$$
\begin{gathered}
\mathfrak{p}=(2, \sqrt{m}) \\
\mathfrak{p}^{6}\left|n, \quad \mathfrak{p}^{3} \| \sqrt{d}, \quad \mathfrak{p}^{3}\right| \mathfrak{M} .
\end{gathered}
$$

Now we set

$$
\beta=\frac{\alpha+1}{2}=\frac{\lambda+\mu}{2 \mu}=\frac{\lambda-\mu+2 \mu}{2 \mu}
$$

then from $\mathfrak{M}\left|\lambda-\mu, \mathfrak{p}^{3}\right| \lambda-\mu$ and $\mathfrak{p}^{2} \| 2 \mu$

$$
\mathfrak{p}^{2} \| \lambda-\mu+2 \mu
$$

holds. Therefore $\beta$ is prime to $\mathfrak{p}$, and for the maximal part $\mathfrak{M}^{\prime}$ of $\mathfrak{M}$ which is prime to 2 , we have as above

$$
\frac{\alpha+1}{2} \equiv 1 \quad\left(\bmod . \mathfrak{M}^{\prime}\right)
$$

Hence $\beta$ is prime to $n$, and especially prime to $d$. And $(\beta) \mathfrak{A}$ is a $\sigma$-invariant ideal of $Q(\sqrt{m})$ prime to $d$, therefore

$$
\beta \mathfrak{A}=(a), \quad a \text { is a rational number prime to } n
$$

holds. Consequently, we have the following proposition:

Proposition $2 "$. Let $m$ be $m \equiv 2$ (mod. 4), $\mathfrak{A}$ an ideal of an ambigous ideal class mod. $\mathfrak{M}$ of $Q(\sqrt{m})$, i.e.

$$
\mathfrak{U}^{\sigma-1}=(\alpha), \quad \alpha \in Q(\sqrt{m}), \alpha \equiv 1(\bmod . \mathfrak{M})
$$

then

$$
\frac{\alpha+1}{2} \mathfrak{A}=(a), \quad \text { a is a rational number prime to } n
$$

and

$$
\mathfrak{A}=\left(\frac{a}{\frac{\alpha+1}{2}}\right) \text { is principal in }(Q \sqrt{m})
$$

Now in consideration of the premises, for any cases we have that $\mathfrak{A}$ is principal in $Q(\sqrt{m})$.

3. An explicit representation for Tannaka-Terada's principal ideal therem. In the following we consider according to three cases of $\mathbf{2}$.

2. I. From the proposition 2 we get 


$$
\mathfrak{A}=\left(\frac{a}{\frac{\alpha+1}{2}}\right), \quad \frac{a}{\frac{\alpha+1}{2}} \equiv a\left(\bmod . \mathfrak{F}_{n}\right)
$$

and, $a$ is a rational number prime to $n$.

Now from S. Takahashi [5], there is an explicit form of an unit which satisfy

$$
a \equiv E(a) \quad\left(\bmod . \mathfrak{F}_{n}\right)
$$

Therefore

$$
\frac{a}{\frac{\alpha+1}{2}} \equiv a \equiv E(a) \quad\left(\bmod . \mathfrak{F}_{n}\right)
$$

and

$$
\mathfrak{A} \sim 1 \quad\left(\bmod . \mathfrak{F}_{n}\right) .
$$

2. II. From the proposition $2^{\prime}$ we get

$$
\mathfrak{U}=\left(\frac{a}{\frac{\alpha+1}{2^{k+1}}}\right)
$$

and if we set $\beta=\frac{a}{\frac{\alpha+1}{2^{k+1}}}$, then $\beta$ is an integer of $Q(\sqrt{m})$ prime to $n$. Now

$$
\beta^{\sigma-1}=(\alpha+1)^{1-\sigma}=\frac{\alpha+1}{\alpha^{\sigma}+1}=\alpha \equiv 1 \quad(\bmod . \mathfrak{M}) .
$$

And if we set $\beta=x+y \sqrt{m}$ ( $x, y$ are rational integers), then from

$$
\begin{gathered}
\beta^{\sigma-1} \equiv 1 \quad(\bmod . \mathfrak{M}), \\
2 y \sqrt{m} \equiv 0 \quad(\bmod . \mathfrak{M})
\end{gathered}
$$

holds. Furthermore, from $\mathfrak{F}_{n} \mid \mathfrak{M}, 2 \| \mathfrak{F}_{n}$

$$
y \sqrt{m} \equiv 0 \quad\left(\bmod . \mathfrak{\mho}_{n} /(2)\right)
$$

holds. On the other hand we have

$$
x \equiv y \quad(\bmod .2) \text {, because } \beta \text { is prime to } \mathfrak{p}=(2,1+\sqrt{m}) .
$$

If $x, y$ are both even, then $2 \mid \beta$, and if $x, y$ are both odd i.e. $x=2 s+1$, $y=2 t+1(s, t$ are rational integers) then 


$$
\beta=2 s+1+(2 t+1) \sqrt{m}=2(s+t \sqrt{m})+(1+\sqrt{m}), \mathfrak{p} \mid \beta .
$$

In the following we consider according to the cases where $x, y$ are even or odd respectively.

i) $x$ : odd, $y:$ even

In this case $y \sqrt{m} \equiv 0$ (mod. 2) holds, so $y \sqrt{m} \equiv 0\left(\bmod . \mathfrak{F}_{n}\right)$ and $\beta=x+y \sqrt{m}$ are prime to $n$ especially prime to $\mathfrak{F}_{n}$, so that $x$ is prime to $\mathfrak{F}_{n}$ and $n$. Therefore

$$
\beta \equiv x \quad\left(\bmod . \mathfrak{F}_{n}\right)
$$

Now from S. Takahashi [5], there is a unit satisfying the congruence equation

$$
x \equiv E(x) \quad\left(\bmod . \mathfrak{F}_{n}\right) .
$$

For this unit we get

$$
\beta \equiv E(x) \quad\left(\bmod . \mathfrak{F}_{n}\right)
$$

and

$$
\mathfrak{A} \sim 1 \quad\left(\bmod . \mathfrak{F}_{n}\right) \quad \text { in } Q\left(\zeta_{n}\right)
$$

ii) $x$ : even, $y:$ odd

It we set $n=2^{t^{\prime}} \cdot n_{0}\left(n_{0}\right.$ : odd), so $x$ is prime to $n$, because $\beta=x+y \sqrt{m}$ is prime to $n$ and $y \sqrt{m} \equiv 0\left(\bmod . \mathfrak{F}_{n} /(2)\right)$. Therefore the following linear congruence equations have the solution $k$, and $k$ relatively prime to $n$

$$
\begin{cases}k x \equiv 1 & \left(\bmod . n_{0}\right) \\ k y \equiv 1 & (\bmod .2)\end{cases}
$$

For this $k$

$$
\left\{\begin{array}{l}
k \beta=k x+k y \sqrt{m} \equiv 1 \quad\left(\bmod . \mathfrak{F}_{n} /(2)\right) \\
k \beta=k x+k y \sqrt{m} \equiv \sqrt{m}(\bmod .2)
\end{array}\right.
$$

hold. Furthermore, from $4 \mid n$

and

$$
i=\sqrt{-1} \in Q\left(\zeta_{n}\right)
$$

$$
\sqrt{m}-i=\frac{1}{i}( \pm \sqrt{-m}+1)
$$

holds. 
On the other hand $\pm \sqrt{-m}+1 / 2$ is an integer, because $-m \equiv 1$ (mod. 4), hence $\sqrt{m} \equiv i$ (mod. 2) holds. So we have from (5) the following congruences,

$$
\begin{cases}k \beta \equiv 1 & \left(\bmod . \mathfrak{F}_{n} /(2)\right) \\ k \beta \equiv i & (\bmod .2)\end{cases}
$$

Let $n=2^{t^{\prime}} \cdot n_{0}=2^{t^{\prime}} \cdot p_{1}^{e_{1}} p_{2}^{e_{2}} \cdots p_{t}^{e_{t}}$ be, where $p_{i}$ are all odd prime numbers and $e_{i} \neq 0$. Then we have

$$
\mathfrak{F}_{n}=2 \mathfrak{F}_{p_{1}} \mathfrak{F}_{p_{2}} \cdots \mathfrak{F}_{p_{t}}, \quad \mathfrak{F}_{p_{t}}=\left(1-\zeta_{p_{t}}\right)
$$

Now we put

$$
\begin{array}{ll}
E_{1}=\prod_{i=1}^{t}\left(1-\zeta_{4} \zeta_{p_{i}}\right), & F_{1}=\prod_{i=1}^{t}\left(\zeta_{p_{i}}-\zeta_{4}\right) \\
E_{2}=\prod_{(i, j)}\left(1-\zeta_{4} \zeta_{p_{i}} \zeta_{p_{j}}\right), & F_{2}=\prod_{(i, j)}\left(\zeta_{p_{i}} \zeta_{p_{j}}-\zeta_{4}\right)
\end{array}
$$

$((i, j)$ : all combinations of two different numbers from $1,2, \cdots, t)$

$$
E_{k}=\prod_{(i, j, \cdots, l)}\left(1-\zeta_{4} \zeta_{p_{t}} \cdots \zeta_{p_{l}}\right), \quad F_{k}=\prod_{(i, j, \cdots, l)}\left(\zeta_{p_{l}} \cdots \zeta_{p_{l}}-\zeta_{4}\right)
$$

$((i, j, \cdots, l):$ all combinations of $k$ difierent numbers from $1,2, \cdots, t)$

$$
E_{t}=1-\zeta_{4} \zeta_{p_{1}} \cdots \zeta_{p_{t}}, \quad F_{t}=\zeta_{p_{1}} \zeta_{p_{2}} \cdots \zeta_{p_{t}}-\zeta_{4}
$$

Then, $E_{1}, E_{2}, \cdots, E_{t}, F_{1}, F_{2}, \cdots, F_{t}$ are units in $Q\left(\zeta_{n}\right)$.

Generally it is well known that if $m$ is a natural number which contains two or more prime numbers, and $\zeta$ is a primitive root of unity, then $1-\zeta$ is a unit. Therefore

$$
\begin{aligned}
& 1-\zeta_{4} \zeta_{p_{t}} \cdots \zeta_{p_{l}} \\
& \zeta_{p_{t}} \zeta_{p_{j}} \cdots \zeta_{p_{l}}-\zeta_{4}=\zeta_{4}\left(\zeta_{4}^{3} \zeta_{p_{t}} \cdots \zeta_{p_{l}}-1\right) \\
& \quad(k=1,2, \cdots, t)
\end{aligned}
$$

are all units. And furthermore, we set 


$$
E= \begin{cases}\frac{E_{1} F_{2} E_{3} F_{4} \cdots E_{t-1} F_{t}}{F_{1} E_{2} F_{3} E_{4} \cdots F_{t-1} E_{t}} & \text { (if } t \text { is even) } \\ \frac{E_{1} F_{2} E_{3} \cdots F_{t-1} E_{t}}{F_{1} E_{2} F_{3} \cdots E_{t-1} F_{t}} & \text { (if } t \text { is odd) }\end{cases}
$$

Then $E$ too is a unit in $Q\left(\zeta_{n}\right)$. Now we put for fixed $i$ from $1,2, \cdots, t$ as follows

$$
\begin{gathered}
E_{1}=E_{1}^{(i)} \bar{E}_{1}^{(i)}, \quad E_{1}^{(i)}=1-\zeta_{4} \zeta_{p_{t}} \\
F_{1}=F_{1}^{(i)} \bar{F}_{1}^{(i)}, \quad F_{1}^{(i)}=\zeta_{p_{t}}-\zeta_{4} \\
\ldots \ldots \ldots \\
E_{k}=E_{k}^{(i)} \bar{E}_{k}^{(i)}, \quad E_{k}^{(i)}=\prod_{(j, \cdots, l)}\left(1-\zeta_{4} \zeta_{i} \cdots \zeta_{l}\right) \\
F_{k}=F_{k}^{(i)} \bar{F}_{k}^{(i)}, \quad F_{k}^{(i)}=\prod_{(j, \cdots, l)}\left(\zeta_{i} \zeta_{j} \cdots \zeta_{l}-\zeta_{4}\right) \\
\cdots \cdots \cdots \\
E_{l}=E_{t}^{(i)} \quad \cdots \cdots \\
F_{t}=F_{t}^{(i)} . \quad\left(\bar{E}_{t}^{(i)}=\bar{F}_{t}^{(i)}=1\right)
\end{gathered}
$$

Then from $\zeta_{p_{i}} \equiv 1\left(\bmod . \mathfrak{F}_{p_{i}}\right)$

$$
\begin{aligned}
& E_{1}^{(i)} \equiv F_{1}^{(i)} \quad\left(\bmod . \mathfrak{F}_{p_{\mathfrak{\imath}}}\right) \\
& E_{k}^{(i)} \equiv \bar{E}_{k-1}^{(i)} \quad\left(\bmod . \mathfrak{F}_{p_{\mathfrak{t}}}\right) \\
& F_{k}^{(i)} \equiv \bar{F}_{k-1}^{(i)} \quad\left(\bmod . \mathfrak{F}_{p_{\mathfrak{t}}}\right) \quad k=2,3, \cdots, t
\end{aligned}
$$

hold. Therefore, from (8), (9), (10)

$$
\begin{gathered}
E=\frac{E_{1}^{(i)} \bar{E}_{1}^{(i)} F_{2}^{(i)} \bar{F}_{2}^{(i)} \cdots}{F_{1}^{(i)} \bar{F}_{1}^{(i)} E_{2}^{(i)} \bar{E}_{2}^{(i)} \cdots} \equiv 1 \quad\left(\bmod . \mathfrak{F}_{p_{\mathfrak{t}}}\right), \\
(i=1,2, \cdots, t) \\
E \equiv 1 \quad\left(\bmod . \mathfrak{F}_{n} /(2)\right) .
\end{gathered}
$$

In the next we show that $E \equiv i(\bmod .2)$. It holds 


$$
\begin{cases}\frac{1-\zeta_{4} \zeta_{p_{i}} \cdots \zeta_{p_{l}}}{\zeta_{p_{i}} \zeta_{p_{j}} \cdots \zeta_{p_{l}}-\zeta_{4}} \equiv \zeta_{4} & (\bmod .2) \\ 1 / \zeta_{4}=-\zeta_{4} \equiv \zeta_{4} & (\bmod .2) .\end{cases}
$$

Therefore

$$
\frac{E_{k}}{F_{k}} \equiv \frac{F_{k}}{E_{k}} \equiv \zeta_{4}^{c_{k}} \quad(\bmod .2)
$$

And from (8) it holds

$$
E \equiv \zeta_{4}^{\sum_{k=1}^{t} t^{c_{k}}}=\zeta_{4}{ }^{(1+1)^{t}-1} \equiv \zeta_{4} \quad(\bmod .2)
$$

Therefore from (11), (12)

$$
\begin{cases}E \equiv 1 & \left(\bmod . \mathfrak{\mho}_{n} /(2)\right) \\ E \equiv i & (\bmod .2)\end{cases}
$$

And from (6) we have

$$
k \beta \equiv E \quad\left(\bmod . \mathfrak{F}_{n}\right) .
$$

Now again take the unit $E(k)$ in $Q\left(\zeta_{n}\right)$ satisfying $k \equiv E(k)\left(\bmod . \mathfrak{F}_{n}\right)$ according to S. Takahashi [5]. Then

$$
\begin{gathered}
\mathfrak{U}=(\beta)=\left(\frac{k \beta}{k}\right) \\
\frac{k \beta}{k} \equiv \frac{E}{E(k)} \quad\left(\bmod . \mathfrak{F}_{n}\right) \\
\mathfrak{U} \sim 1 \quad\left(\bmod . \mathfrak{F}_{n}\right) \text { in } Q\left(\zeta_{n}\right) .
\end{gathered}
$$

2. III From the proposition 2 " we have

$$
\mathfrak{U}=\left(\frac{a}{\frac{\alpha+1}{2}}\right)
$$

and put

$$
\beta=\frac{a}{\frac{\alpha+1}{2}}
$$

$\beta$ is an integer of $Q(\sqrt{m})$ which is prime to $n$, and 


$$
\beta^{\sigma-1}=(\alpha+1)^{1-\sigma}=\alpha \equiv 1 \quad(\bmod . \mathfrak{M})
$$

Therefore if we put

$$
\beta=x+y \sqrt{m} \quad(x, y \text { are rational integers })
$$

then

$$
\beta^{\sigma-1}=\frac{x-y \sqrt{m}}{x+y \sqrt{m}}-1=\frac{-2 y \sqrt{m}}{x+y \sqrt{m}} \equiv 0(\bmod . \mathfrak{M})
$$

So

$$
2 y \sqrt{m} \equiv 0 \quad(\bmod . \mathfrak{M})
$$

and $x$ is prime to $n$

$$
y \sqrt{m} \equiv 0 \quad\left(\bmod . \mathfrak{F}_{n} /(2)\right)
$$

In the following we consider according to the cases where $y$ is even or odd respectively.

i) $y:$ even

In this case it holds from (14),

$$
y \sqrt{m} \equiv 0\left(\bmod . \mathfrak{F}_{n}\right),
$$

so

$$
\beta \equiv x \quad\left(\bmod . \mathfrak{F}_{n}\right)
$$

and $x$ is prime to $n$. Therefore take again a unit in $Q\left(\zeta_{n}\right)$ satisfying $x \equiv E(x)$ $\left(\bmod . \mathfrak{F}_{n}\right)$. Then

$$
\mathfrak{U}=(\beta), \beta \equiv x \equiv E(x) \quad\left(\bmod . \mathfrak{F}_{n}\right)
$$

so it holds

$$
\mathfrak{A} \sim 1\left(\bmod . \mathfrak{F}_{n}\right) \text { in } Q\left(\zeta_{n}\right)
$$

ii) $y$ : odd

Write $n=2^{t} \cdot n_{0}, n_{0}$ being odd. Then the following linear congruence equations have the solution $k$ which is prime to $n$

$$
\left\{\begin{aligned}
k x \equiv 1 & \left(\bmod . n_{0}\right) \\
k \equiv 1 & (\bmod .2)
\end{aligned}\right.
$$


so, for $\beta=x+y \sqrt{m}$

$$
\left\{\begin{array}{l}
k \beta=k x+k y \sqrt{m} \equiv 1+\sqrt{m} \quad(\bmod .2) \\
k \beta=k x+k y \sqrt{m} \equiv 1 \quad\left(\bmod . \mathfrak{F}_{n} /(2)\right)
\end{array}\right.
$$

hold. (phr. $\mathfrak{F}_{n} /(2) \mid n_{0}, y \sqrt{m} \equiv 0\left(\bmod . \mathfrak{F}_{n} /(2)\right)$.

Now we write $m=2 m^{\prime}\left(m^{\prime}\right.$ is odd). Here, if $m^{\prime} \equiv 1(\bmod .4)$ holds

$$
\sqrt{m}-\sqrt{2}=\sqrt{2}\left(\sqrt{m^{\prime}}-1\right) \text { in } Q\left(\zeta_{n}\right)
$$

and $\sqrt{m^{\prime}}-1 / 2$ is an integer in $Q\left(\sqrt{m^{\prime}}\right) \subset Q\left(\zeta_{n}\right)$

so

$$
\sqrt{m} \equiv \sqrt{2}(\bmod .2)
$$

And if $m^{\prime} \equiv 3$ (mod. 4) holds

$$
\sqrt{m}-\sqrt{2} i=\sqrt{2}\left(\sqrt{m^{\prime}}-i\right)
$$

and $\sqrt{m^{\prime}}-i / 2=\frac{1}{2 i} \cdot\left( \pm \sqrt{-m^{\prime}}+1\right)$ is an integer, because $-m^{\prime} \equiv 1(\bmod .4)$

so

$$
\sqrt{m} \equiv \sqrt{2} i \quad(\bmod .2)
$$

On the other hand take

$$
\zeta_{8}=\frac{1+i}{\sqrt{2}} \in Q\left(\zeta_{8}\right)
$$

then

$$
\begin{aligned}
\zeta_{8}+\zeta_{8}^{-1} & =\frac{1+i}{\sqrt{2}}+\frac{\sqrt{2}}{1+i} \\
& =\frac{\sqrt{2}(1+i)}{2}+\frac{\sqrt{2(1-i)}}{2} \\
& =\sqrt{2} .
\end{aligned}
$$

Therefore

$$
\begin{aligned}
\sqrt{2}-\sqrt{2 i} & =\sqrt{2}(1-i)=\left(\zeta_{8}+\zeta_{8}^{-1}\right)\left(1-\zeta_{8}^{2}\right) \\
& =\zeta_{8}+\zeta_{8}^{-1}-\zeta_{8}^{3}-\zeta_{8} \\
& =\zeta_{8}^{-1}\left(1-\zeta_{8}^{4}\right)=2 \zeta_{8}^{-1} \equiv 0(\bmod .2)
\end{aligned}
$$

From the above we have for any cases

$$
1+\sqrt{m} \equiv 1+\sqrt{2} \quad(\bmod .2) .
$$


Now $1+\sqrt{2}$ is a unit in $Q\left(\zeta_{n}\right)$, and has the following representasion.

$$
\begin{aligned}
1+\sqrt{2} & =1+\zeta_{8}+\zeta_{8}^{-1}, \quad \zeta_{8}^{4}=-1, \quad \zeta_{8}^{3}=-\zeta_{8}^{-1} \\
& =1+\zeta_{8}-\zeta_{8}^{3} .
\end{aligned}
$$

On the other hand

$$
\begin{aligned}
\left(1+\zeta_{8}-\zeta_{8}^{3}\right)\left(1-\zeta_{8}\right) & =1+\zeta_{8}-\zeta_{8}^{3}-\zeta_{8}-\zeta_{8}^{2}+\zeta_{8}^{4} \\
& =-\zeta_{8}^{3}-\zeta_{8}^{2}
\end{aligned}
$$

hence

$$
1+\zeta_{8}-\zeta_{8}^{3}=\frac{\zeta_{8}^{3}+\zeta_{8}^{2}}{\zeta_{8}-1}=\frac{\zeta_{8}^{3}+\zeta_{4}}{\zeta_{8}-1}
$$

In the following we write

$$
E_{0}=1+\sqrt{2}=\frac{\zeta_{8}^{3}+\zeta_{4}}{\zeta_{8}-1}
$$

so from (15)

$$
\left\{\begin{array}{l}
k \beta \equiv E_{0}(\bmod .2) \\
k \beta \equiv 1 \quad\left(\bmod . \mathfrak{F}_{n} /(2)\right) .
\end{array}\right.
$$

Now let all prime numbers contained in $n_{0}$ be $p_{1}, p_{2}, \cdots, p_{t}$, and we put

$$
\begin{aligned}
& E_{1}=\prod_{i=1}^{t} \frac{\zeta_{8}-\zeta_{p_{t}}}{\zeta_{8}^{3}+\zeta_{4} \zeta_{p_{t}}} \cdot \frac{\zeta_{4} \zeta_{p_{t}}-1}{\zeta_{4}-\zeta_{p_{t}}} \\
& E_{2}=\prod_{(i, j)} \frac{\zeta_{8}-\zeta_{p_{i}} \zeta_{p_{j}}}{\zeta_{8}^{3}+\zeta_{4} \zeta_{p_{t}} \zeta_{p_{j}}} \cdot \frac{\zeta_{4} \zeta_{p_{t}} \zeta_{p_{j}}-1}{\zeta_{4}-\zeta_{p_{t}} \zeta_{p_{j}}}
\end{aligned}
$$

$((i, j)$ : all combinations of two different numbers from $1,2, \cdots, t)$

$$
E_{k}=\prod_{\left(i, j_{1} \cdots, l\right)} \frac{\zeta_{8}-\zeta_{p_{t}} \zeta_{p_{j}} \cdots \zeta_{p_{l}}}{\zeta_{8}^{3}+\zeta_{4} \zeta_{p_{t}} \cdots \zeta_{p_{l}}} \cdot \frac{\zeta_{4} \zeta_{p_{i}} \cdots \zeta_{p_{l}}-1}{\zeta_{4}-\zeta_{p_{t}} \zeta_{p_{j}} \cdots \zeta_{p_{l}}}
$$

$((i, j, \cdots, l)$ : all combinations of $k$ different numbers from $1,2, \cdots, t)$

$$
E_{t}=\frac{\zeta_{8}-\zeta_{p_{1}} \zeta_{p_{2}} \cdots \zeta_{p_{t}}}{\zeta_{8}^{3}+\zeta_{4} \zeta_{p_{1}} \cdots \zeta_{p_{t}}} \cdot \frac{\zeta_{4} \zeta_{p_{i}} \cdots \zeta_{p_{l}}-1}{\zeta_{4}-\zeta_{p_{t}} \zeta_{p_{j}} \cdots \zeta_{p_{l}}}
$$


Now

$$
\begin{aligned}
& \zeta_{8}-\zeta_{p_{t}} \zeta_{p_{s}} \cdots \zeta_{p_{l}}=\zeta_{8}\left(1-\zeta_{8}^{7} \zeta_{p_{t}} \cdots \zeta_{p_{l}}\right) \\
& \zeta_{8}^{3}+\zeta_{4} \zeta_{p_{t}} \cdots \zeta_{p_{l}}=\zeta_{8}^{3}\left(1-\zeta_{8}^{3} \zeta_{p_{t}} \cdots \zeta_{p_{l}}\right) \\
& \zeta_{4} \zeta_{p_{t}} \cdots \zeta_{p_{l}}-1 \\
& \zeta_{4}-\zeta_{p_{t}} \zeta_{p} \cdots \zeta_{p_{l}}=\zeta_{4}\left(1-\zeta_{4}^{3} \zeta_{p_{i}} \cdots \zeta_{p_{l}}\right)
\end{aligned}
$$

are all units. Therefore $E_{1}, E_{2}, \cdots, E_{t}$ are all units in $Q\left(\zeta_{n}\right)$. Now, for fixed $i$ from $1,2, \cdots, t$ we put

$$
\begin{gathered}
E_{1}=E_{1}^{(i)} \bar{E}_{1}^{(i)}, \quad E_{1}^{(i)}=\frac{\zeta_{8}-\zeta_{p_{t}}}{\zeta_{8}^{3}+\zeta_{4} \zeta_{p_{t}}} \cdot \frac{\zeta_{4} \zeta_{p_{t}}-1}{\zeta_{4}-\zeta_{p_{t}}} \\
E_{2}=E_{2}^{(i)} \bar{E}_{2}^{(i)}, \quad E_{2}^{(i)}=\prod_{j} \frac{\zeta_{8}-\zeta_{p_{t}} \zeta_{p_{j}}}{\zeta_{8}^{3}+\zeta_{4} \zeta_{p_{t}} \zeta_{p_{j}}} \cdot \frac{\zeta_{4} \zeta_{p_{t}} \zeta_{p_{j}}-1}{\zeta_{4}-\zeta_{p_{t}} \zeta_{p_{s}}} \\
\cdots \cdots \cdots \cdots \\
E_{k}=E_{k}^{(i)} \bar{E}_{k}^{(i)}, \quad E_{k}^{(i)}=\prod_{(j, \cdots, l)} \frac{\zeta_{8}-\zeta_{p_{t}} \zeta_{p_{j}} \cdots \zeta_{p_{l}}}{\zeta_{8}^{3}+\zeta_{4} \zeta_{p_{t}} \cdots \zeta_{p_{l}}} \cdot \frac{\zeta_{4} \zeta_{p_{t}} \cdots \zeta_{p_{l}}-1}{\zeta_{4}-\zeta_{p_{t}} \zeta_{p} \cdots \zeta_{p_{l}}} \\
\cdots \cdots \cdots \\
E_{t}=E_{t}^{(i)} .
\end{gathered}
$$

Then

$$
\begin{aligned}
E_{0} E_{1}^{(i)} & =\frac{\zeta_{8}^{3}+\zeta_{4}}{\zeta_{8}-1} \cdot \frac{\zeta_{8}-\zeta_{p_{t}}}{\zeta_{8}^{3}+\zeta_{4} \zeta_{p_{t}}} \cdot \frac{\zeta_{4} \zeta_{p_{i}}-1}{\zeta_{4}-\zeta_{p_{t}}} \\
& \equiv \frac{\zeta_{8}^{3}+\zeta_{4}}{\zeta_{8}-1} \cdot \frac{\zeta_{8}-1}{\zeta_{8}^{3}+\zeta_{4}} \cdot \frac{\zeta_{4}-1}{\zeta_{4}-1} \\
& =1 \quad\left(\bmod . \mathfrak{\digamma}_{p_{t}}\right)
\end{aligned}
$$

and for $k=2,3, \cdots, t$

$$
\begin{aligned}
E_{k}^{(i)} & =\prod_{(j \cdots l)} \frac{\zeta_{8}-\zeta_{p_{l}} \zeta_{p_{j}} \cdots \zeta_{p_{l}}}{\zeta_{8}^{3}+\zeta_{4} \zeta_{p_{t}} \cdots \zeta_{p_{l}}} \cdot \frac{\zeta_{4} \zeta_{p_{1}} \cdots \zeta_{p_{l}}-1}{\zeta_{4}-\zeta_{p_{l}} \zeta_{p_{j}} \cdots \zeta_{p_{l}}} \\
& \equiv \prod_{(j \cdots l)} \frac{\zeta_{8}-\zeta_{p_{j}} \cdots \zeta_{p_{l}}}{\zeta_{8}^{3}+\zeta_{4} \zeta_{p_{j}} \cdots \zeta_{p_{l}}} \cdot \frac{\zeta_{4} \zeta_{p_{j}} \cdots \zeta_{p_{l}}-1}{\zeta_{4}-\zeta_{p} \cdots \zeta_{p_{l}}} \\
& =\bar{E}_{k-1}^{(i)} \quad\left(\bmod . \Im_{p_{l}}\right)
\end{aligned}
$$

holds. Therefore we have the following congruence equations 


$$
\left\{\begin{array}{c}
E_{0} E_{1}^{(i)} \equiv 1 \quad\left(\bmod . \mathfrak{F}_{p_{t}}\right) \\
E_{k}^{(i)} \equiv \bar{E}_{k-1}^{(i)}\left(\bmod . \widetilde{F}_{p_{t}}\right) \\
(k=2,3, \cdots, t) .
\end{array}\right.
$$

Now if $t$ is even we put

$$
\begin{aligned}
E & =\frac{E_{0} E_{1} E_{3} \cdots E_{t-1}}{E_{2} E_{4} \cdots E_{t}} \\
& =\frac{E_{0} E_{1}^{(i)} \bar{E}_{1}^{(i)} \cdots E_{t-1}^{(i)} \bar{E}_{t-1}^{(i)}}{E_{2}^{(i)} \bar{E}_{2}^{(i)} E_{4}^{(i)} \bar{E}_{4}^{(i)} \cdots E_{t}^{(i)}}
\end{aligned}
$$

So from (17)

$$
E \equiv 1 \quad\left(\bmod . \mathfrak{F}_{p_{\mathfrak{t}}}\right)
$$

If $t$ is odd we put

$$
\begin{aligned}
E & =\frac{E_{0} E_{1} E_{3} \cdots E_{t}}{E_{2} E_{4} \cdots E_{t-1}} \\
& =\frac{E_{0} E_{1}^{(i)} \bar{E}_{1}^{(i)} \cdots E_{t}^{(i)}}{E_{2}^{(i)} \bar{E}_{2}^{(i)} \cdots E_{t-1}^{(i)} \bar{E}_{t-1}^{(i)}}
\end{aligned}
$$

So from (17) we have

$$
E \equiv 1 \quad\left(\bmod . \mathfrak{F}_{p_{\mathfrak{t}}}\right)
$$

As the above we have for any cases

$$
E \equiv 1 \quad\left(\bmod . \mathfrak{F}_{p_{i}}\right) i=1,2, \cdots, t,
$$

accordingly

$$
E \equiv 1 \quad\left(\bmod . \mathfrak{F}_{n} /(2)\right)
$$

On the other hand we can show that $E \equiv E_{0}$ (mod. 2).

Put for brevity as the following

$$
\begin{aligned}
& B=\zeta_{p_{t}} \zeta_{p_{s}} \cdots \zeta_{p_{l}} \\
& A=\frac{\zeta_{8}-\zeta_{p_{t}} \zeta_{p_{s}} \cdots \zeta_{p_{l}}}{\zeta_{8}^{3}+\zeta_{4} \zeta_{p_{t}} \cdots \zeta_{p_{l}}} \cdot \frac{\zeta_{4} \zeta_{p_{t}} \cdots \zeta_{p_{l}}-1}{\zeta_{4}-\zeta_{p_{t}} \zeta_{p^{\prime}} \cdots \zeta_{p_{l}}}
\end{aligned}
$$

Then 


$$
\begin{aligned}
A-1 & =\frac{\zeta_{8}-B}{\zeta_{8}^{3}+\zeta_{4} B} \cdot \frac{\zeta_{4} B-1}{\zeta_{4}-B}-1 \\
& =\frac{\left(\zeta_{8}-B\right)\left(\zeta_{4} B-1\right)-\left(\zeta_{8}^{3}+\zeta_{4} B\right)\left(\zeta_{4}-B\right)}{\left(\zeta_{8}^{3}+\zeta_{4} B\right)\left(\zeta_{4}-B\right)} \\
& =\frac{\zeta_{8} \zeta_{4} B-\zeta_{8}-\zeta_{4} B^{2}+B-\zeta_{8}^{3} \zeta_{4}+\zeta_{8}^{3} B-\zeta_{4}^{2} B+\zeta_{4} B^{2}}{\left(\zeta_{8}^{3}+\zeta_{4} B\right)\left(\zeta_{4}-B\right)} \\
& =\frac{2 B\left(1+\zeta_{8}^{3}\right)}{\left(\zeta_{8}^{3}+\zeta_{4} B\right)\left(\zeta_{4}-B\right)} \equiv 0 \quad(\bmod .2) .
\end{aligned}
$$

Namely

$$
A \equiv 1 \quad(\bmod .2)
$$

and so

$$
\left\{\begin{array}{l}
E_{1} \equiv E_{2} \equiv \cdots \equiv E_{t} \equiv 1(\bmod .2) \\
E \equiv E_{0} \quad(\bmod .2) .
\end{array}\right.
$$

From the above formulas (16), (18), (19)

$$
k \beta \equiv E \quad\left(\bmod . \mathfrak{F}_{n}\right) .
$$

Now take once again according to S. Takahashi [5] a unit $E(k)$ in $Q\left(\zeta_{n}\right)$ satisfying the congruence equation $k \equiv E(k)\left(\bmod . \mathfrak{F}_{n}\right)$, then we have the following congruence which is the desired result:

$$
\begin{aligned}
& \beta=\frac{k \beta}{k} \equiv \frac{E}{E(k)} \quad\left(\bmod . \mathfrak{F}_{n}\right) \\
& \mathfrak{A} \sim 1 \quad\left(\bmod . \mathfrak{F}_{n}\right) \text { in } Q\left(\zeta_{n}\right) .
\end{aligned}
$$

\section{REFERENCES}

[1] T. TANNAKA, A generalised principal ideal theorem and a proof of a conjecture of Deuring, Ann. of Math., 67(1958).

[2] F. TERAdA, On a generalization of the principal ideal theorem, Tôhoku Math. Journ., (2) $1(1949)$.

[3] T. TANNAKA, An alternative proof of a generalized principal ideal theorem, Proc. Japan Acad., 25(1949).

[4] - On the generalized principal ideal theorem, Proc. of the international symposium on algebraic number theory, Tokyo-Nikoo, 1955.

[5] S. TAKAHASHI, An explicit representation of the generalized principal ideal theorem for the rational ground field, Tôhoku Math. Journ., 16,2(1964).

MATH. INSTITUTE

IWATE UNIVERSITY. 